\title{
Reinstatement of Cue-induced Methamphetamine-seeking Behaviour
}

\author{
Baomiao Ma ${ }^{\text {a }}$, Kai Yue ${ }^{\mathrm{a}}$, Junqiao Xing ${ }_{\mathrm{a}}^{\mathrm{a}}$, Xiaokang Gong ${ }^{\mathrm{a}}$, Lin Chen ${ }^{\mathrm{a}}$, Qin Ru \\ Qi Xiong ${ }^{a}$, Xiang Tian ${ }^{a}$, Rihui Wu ${ }^{a}$, Yongping Gan ${ }^{b}$, Daisong Wang ${ }^{b}$, \\ Chaoying $\mathrm{Li}^{\mathrm{a}, *}$ \\ ${ }^{a}$ Wuhan Institutes of Biomedical Sciences, Jianghan University, Wuhan 430056, China \\ ${ }^{b}$ Drug Prevention and Education Center, Hubei Public Security Bureau, Wuhan 430070, China \\ *Corresponding authors: email: licy.whibs.corresp@foxmail.com
}

Keywords: Methamphetamine, Self-administration, Relapse, Cue-induced, extinction, Animal model.

\begin{abstract}
Cue-induced methamphetamine (METH) craving increases after prolonged forced abstinence from METH. In the present study, we employed male Sprague-Dawley rats to study METH reinstatement behavior induced by conditioned cue. We trained rats to self-administer METH under a fixed ratio 1 schedule for 10 sessions (4 hours/day), and then assessed METH induced by conditioned cues after $12 \mathrm{~d}$ extinction. Under both training and extinction conditions, the animals showed increased responding of nose poking on cue-induced during the period reinstatement. The results suggested that self-administration responding could be reinitiated by cue-induced from the drug environment in rats. The procedure used in this model may be useful for the study of the underlying mechanism of cue-induced reinstatement.
\end{abstract}

\section{Introduction}

Methamphetamine (METH) is a highly potent and addictive psychostimulant with severe detrimental effects to the health of users. Relapse to METH-seeking behavior is a primary manifestation of drug addiction, and reducing relapse is a clinical index of the success of interventions $^{[1]}$. Nevertheless, there are currently no approved pharmacotherapies for directly treating METH abuse, nor any to complement the limited behavioral interventions ${ }^{[2]}$.

Relapse to drug taking after successful abstinence is one of the primary challenges to the successful treatment of addiction. Potential relapse pharmacotherapies are often evaluated preclinically using the operant reinstatement paradigm ${ }^{[3]}$. The reinstatement model is widely used to study relapse to drug addiction. In the self-administration version of the reinstatement model,which is based on an operant-conditioning paradigm, animals are trained to respond for drug infusions, following extinction of the responding, at least three major classes of events are believed play a role in promoting relapse: re-exposure to a single dose of the previously self-administered drug or a related drug (priming) ${ }^{[4,5]}$, re-exposure to environmental stimuli which have been paired with drug use (cues) ${ }^{[6]}$ and stressors ${ }^{[7]}$. The majority of the reinstatement literature to date has focused on cocaine, opioids and ethanol. Considerably less attention has been devoted to METH. The aim of the current study was to establish a rat model of METH relapse behavior induced by conditioned cue.

\section{Materials and methods}

Animals. Sixteen male Sprague-Dawley rats (Animal Center of the Tongji Medical College of Huazhong University of Science \& Technology, Wuhan, China), weighing approximately 250-275 g, 
were individually housed upon arrival. Animals were maintained on a $12 \mathrm{hr}$ light-dark cycle (lights onset 21:00 h, offset 09:00 h) in a temperature and humidity controlled rodent colony. Food and water were freely available except when specified. All experimental procedures followed the guidelines of the Principles of Laboratory Animal Care (National Institutes of Health publication number 86-23, 1996).

Drugs. METH was obtained from the Hubei Public Security Bureau and was dissolved in heparinized ( 5 units $/ \mathrm{ml}$ ) sterile saline for the intravenous self-administration solution. METH for i.p. priming was diluted in sterile saline to a volume of $1 \mathrm{ml} / \mathrm{kg}$ of body weight.

Surgery. Prior to self-administration training, rats were prepared with intravenous catheters into the jugular vein ${ }^{[8]}$. Briefly, rats were anesthetized with sodium pentobarbital $(50 \mathrm{mg} / \mathrm{kg}$, i.p.). Atropine sulfate and penicillin B were given at the time of surgery. All rats were implanted with chronically indwelling i.v. catheters. A silicon catheter $(3.5 \mathrm{~cm}$ in length, $0.58 \mathrm{~mm}$ in inner diameter, $0.91 \mathrm{~mm}$ in outer diameter; BPU-T30, Instech, Plymouth Meeting, PA, USA) was inserted into the right external jugular vein so that the tip reached the right atrium and was secured with thread. The other end of the catheter $(10 \mathrm{~cm}$, PE20) was passed s.c. through an incision on the back of the body, where it exited into a custom-made fluid-connector fixed to a jacket. The catheters were flushed daily with $0.2 \mathrm{ml}$ saline-heparin solution $(25 \mathrm{U} / \mathrm{ml}$ heparin) to maintain catheter patency. Following catheter surgery, each rat was housed individually in its home cage and was allowed five to seven days of recovery during which it received a daily intravenous infusion of gentamicin $(0.16 \mathrm{mg} / \mathrm{kg})$ followed by $0.2 \mathrm{ml}$ of a heparinized (1\%) sterile saline solution to flush the antibiotic through the catheter.

METH self-administration. Rats were trained to self-administer METH during daily $4 \mathrm{~h}$ sessions under a fixed ratio 1 schedule of reinforcement. Rats received a single METH infusion (0.06 $\mathrm{mg} / \mathrm{kg}$ )) following an active nose-poke. Each infusion was paired with a $5 \mathrm{~s}$ illumination of a light in combination with the noise of the infusion pump; together these stimuli served as a discrete conditioned cue paired with the drug infusion. Following infusions, a time-out period was imposed for $20 \mathrm{~s}$, during which responding was recorded but produced no programmed consequences. Rats were put back into their individual home cages shortly after the session. An acquisition criterion required that subjects' active nose-pokes vary by $\leq 10 \%$ over the course of three consecutive maintenance days.

Extinction Procedures. Extinction sessions were $2 \mathrm{hr}$ in length and commenced following stabilization of self-administration. During the extinction session, the rats were brought to the self-administration chambers in the absence of METH infusions and the previously relevant cues. The extinction criterion was that the last active responses are less than $10 \%$ of the average responding on the active nose-poke during maintenance.

Cues-induced reinstatement Procedures. A single $4 \mathrm{hr}$ reinstatement test session was conducted on the day following the 12 extinction session. In the reinstatement induced by cues, the discrete Conditioned stimulus (CS) were presented for $5 \mathrm{~s}$, after which each active nosepoke response resulted in another presentation of the CS. Nose-pokes during this phase of CS reinstatement were accumulated for over $240 \mathrm{~min}$.

Statistical analysis. The data were expressed as the mean \pm SEM. The differences in total active responses, inactive responses and locomotor activity were analyzed by one-way analyses of variance (ANOVA) followed by the least significant difference (LSD) post hoc test. All statistical analyses were performed by with SPSS for Windows, version 11.5 (SPSS Inc., Chicago, IL, USA). The level of significance was set at $\mathrm{p}<0.05$.

\section{Results}

The acquisition of METH self-administration. Fig. 1 shows the average number of the active and inactive nose pokes for all rats that were tested. Rats acquired METH $(0.06 \mathrm{mg} / \mathrm{kg} / \mathrm{infusion})$ self-administration and responding was stable for the last 3 days of training on an FR1 schedule of reinforcement, There was no significant difference in either average responding or intake of METH 
self-administration during the last three days of the maintenance phase. The animals exhibited reliable self-administration of METH with (70.4 \pm 1.7$)$ active nose-poke responses per session.

\section{Training}

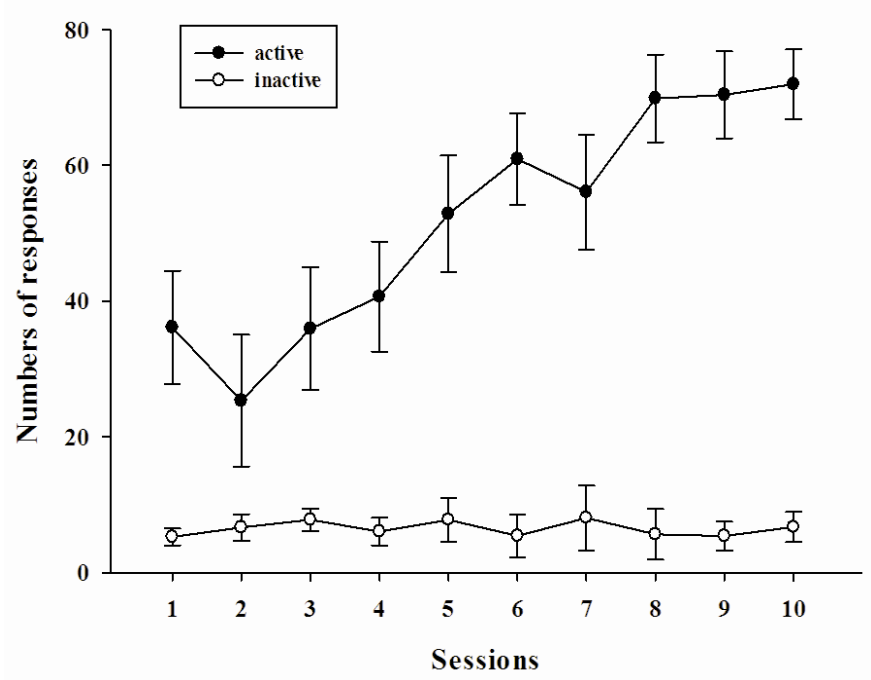

Fig. 1 The acquisition of METH self-administration. Active and inactive responses (nose pokes) during METH self-administration over 10 consecutive days (mean \pm S.E.M.).

Cue-induced drug seeking. Fig. 2 shows the mean \pm S.E.M. number of nose-pokes on the active and inactive nose-pokes during the 12 days of extinction. All of the animals exhibited significantly diminished responding over time. Following extinction, The 4-h relapse tests were conducted; active responses on the Cue-induced reinstatement test was comparable to the end of training. Cue-induced response occurred mainly in the previous METH-associated active nose-pokes, with total 4-h responses of $(76.3 \pm 1.9)$. Responding at the inactive nose-poke was minimal during both testing phases.

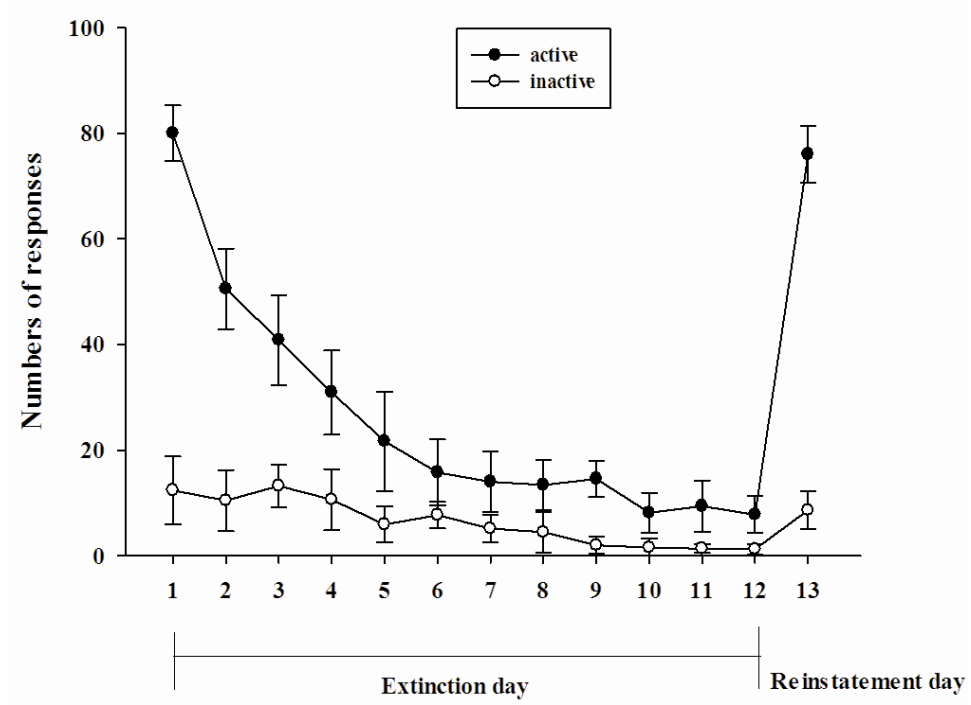

Fig. 2. Mean $( \pm$ SEM) active and inactive responses on extinction and cue-induced reinstatement testing. 


\section{Conclusion}

On the basis of the present data, it appears that, for cue-induced behavioral interventions to succeed, and suggest conditions environmentally and pharmacologically likely more similar to relapse in the drug abuser which should be considered during the evaluation of potential pharmacotherapies.

\section{Acknowledgement}

This work was supported by the National Natural Science Foundation of China (81302762). No potential conflicts of interest were disclosed.

\section{References}

[1] O'Brien CP, Gardner EL. Critical assessment of how to study addiction and its treatment: human and non-human animal models. Pharmacol Ther. 2005. 108(1): 18-58.

[2] Obert JL, McCann MJ, Marinelli-Casey P, et al. The matrix model of outpatient stimulant abuse treatment: history and description. J Psychoactive Drugs. 2000. 32(2): 157-64.

[3] Shaham Y, Shalev U, Lu L, De Wit H, Stewart J. The reinstatement model of drug relapse: history, methodology and major findings. Psychopharmacology (Berl). 2003. 168(1-2): 3-20.

[4] Zhou W, Zhang F, Tang S, Liu H, Gu J, Yang G. The dissociation of heroin-seeking patterns induced by contextual, discriminative, or discrete conditioned cues in a model of relapse to heroin in rats. Psychopharmacology (Berl). 2005. 181(2): 197-206.

[5] de Wit H, Stewart J. Reinstatement of cocaine-reinforced responding in the rat. Psychopharmacology (Berl). 1981. 75(2): 134-43.

[6] Diergaarde L, de Vries W, Raaso H, Schoffelmeer AN, De Vries TJ. Contextual renewal of nicotine seeking in rats and its suppression by the cannabinoid-1 receptor antagonist Rimonabant (SR141716A). Neuropharmacology. 2008. 55(5): 712-6.

[7] Erb S, Shaham Y, Stewart J. Stress reinstates cocaine-seeking behavior after prolonged extinction and a drug-free period. Psychopharmacology (Berl). 1996. 128(4): 408-12.

[8] Zhou W, Zhang F, Liu H, et al. Effects of training and withdrawal periods on heroin seeking induced by conditioned cue in an animal of model of relapse. Psychopharmacology (Berl). 2009. 203(4): 677-84. 